\title{
A Fuzzy Predictive PID Control Scheme for the Excitation System of Synchronous Generator
}

\author{
Yang Zheng ${ }^{1}$, Jianzhong Zhou ${ }^{1, a}$, Chu Zhang ${ }^{1}$, Yuncheng Zhang ${ }^{1}$ and Wei Zhao ${ }^{1}$ \\ ${ }^{1}$ School of Hydropower and Information Engineering, Huazhong University of Science and Technology, Wuhan, \\ Hubei 430074, P R China
}

\begin{abstract}
With the rapid development of the process control theories in the electrical engineering, new control strategies which lead to better performances are urgently demanded for the excitation control of synchronous generators. For the sake of improving the convergence rate of the terminal voltage and covering the weakness in the adaptability of operational conditions of conventional controls in disturbances, a fuzzy predictive PID excitation control method is proposed in this paper. This control scheme can be divided into three steps in every sample interval: PID parameter adaptation, rolling state prediction and realtime control movement integration. Numerical simulations have been conducted under different operational conditions with the proposed method as well as the conventional ones, respectively. Experimental comparisons indicate the superiority in voltage regulation performance of the proposed method.
\end{abstract}

\section{Introduction}

Although PID control is still being applied in most excitation system of synchronous generators due to its robustness in control and simplicity in design, its deficiencies including dependence on control parameters, weakness in condition adaptation and hardship of parameter tuning make scholars worldwide find ways to improve its performance or design the new control methods with better performance. For the sake of improving the condition adaptation characteristic of PID, parameter tuning tactics such as the swarm intelligence optimization [1], fuzzy logic [2], artificial neural network [3] and their comprehension [4] have been extensively studied over the decades. These efforts do provide us with approaches to make PID parameters more closely match the working conditions in excitation control and to attenuate the deterioration in operating mode transitions. However, these aforementioned methods cannot get rid of the intrinsic defects of PID control, namely, it lacks the ability of prediction. To this end, some scholars have brought the notion of model predictive control into PID control. For instance, Zhang proposed a predictive control optimization based PID control for industrial surfactant reactors described by linear state-space equations [5] and Sato built a GPC-based PID controller for a weigh feeder [6], etc. Most of these predictive integrated PID control methods are either designed for linear systems or possess heavy calculation burden for the inevitably online rolling optimization process [7], thus the application of these control schemes still have many limitations.

\footnotetext{
${ }^{a}$ Corresponding Author: jz.zhou@hust.edu.cn 
This paper presents a modified fuzzy predictive PID (FP-PID) control scheme in which the control signal takes account of not only the past and present error of the system output, but also the future predictive state information. In the design of this predictive controller, rolling optimization process in every sampling instance is replaced by a rolling state prediction process based on fuzzy PID control with much lower calculation burden. Confidence probability is introduced to the real-time control signal integration with the predictive control movement vector recording from rolling state prediction, rather than implementing only the first element of the control sequence like ordinary predictive control methods. Numerical simulations have been conducted under voltage step and three-phase short circuit conditions in a power system with a single machine connected to an infinite bus. Simulation results validate the enhancement of the FP-PID control in voltage-tracking performance compared with PID and fuzzy PID controls.

The remainder of the paper is organized as follows: design of the FP-PID control strategy in affine nonlinear systems is explained in detail in Section 2. Then, nonlinear models for the excitation system is stated in Section 3. Section 4 discusses the numerical simulations with the utility of the proposed control scheme and the comparative ones. Eventually, conclusions are summarized in Section 5.

\section{Fuzzy Predictive PID Control for Nonlinear Systems}

The fuzzy predictive PID (FP-PID) control scheme is designed on the basis of discrete-time affine nonlinear systems that can be described in Equation (1).

$$
\left\{\begin{array}{l}
\mathbf{X}(\mathrm{k}+1)=\mathrm{f}(\mathbf{X}(\mathrm{k}))+\mathrm{g}(\mathrm{k}) \cdot u(\mathrm{k}) \\
y(\mathrm{k})=\mathrm{h}(\mathbf{X}(\mathrm{k}))
\end{array}\right.
$$

where, $\mathrm{k}$ is the sample number, $\mathrm{X}$ represents the state vector, $\mathrm{u}$ is the input variable, $\mathrm{y}$ is the system output.

\subsection{Adaptive control Parameters Calculation Using Fuzzy Logic}

At every sample time, the real-time PID parameters are updated by fuzzy logic methods so as to make the controller adapt the current working condition of the system. The fuzzy parameter tuning is similar to the way in the extensive studied literature about fuzzy PID control and is briefly stated as follows:

(1) Build fuzzy rule tables of the three PID parameter increments (i.e., $\Delta K_{p}, \Delta K_{i}, \Delta K_{d}$ ) according to the variation characteristics of the parameters, respectively. Both of the fuzzy sets of error and error deviation are $[N B, N M, N S, Z O, P S, P M, P B]$. The fuzzy membership is conducted with triangular function.

(2) Calculate the fuzzy subset and the fuzzy membership of each subset of $\Delta K_{p}, \Delta K_{i}, \Delta K_{d}$ with the current output deviation and its increment as the fuzzy reasoning machine.

(3) Determine the value of $\Delta K_{p}, \Delta K_{i}, \Delta K_{d}$ by the defuzzination with Center of Gravity Method.

(4) Calculate the current parameters $K_{p}, K_{i}, K_{d}$ by adding the calculated $\Delta K_{p}, \Delta K_{i}, \Delta K_{d}$ to the initial parameters $K_{p 0}, K_{i 0}, K_{d 0}$.

\subsection{Fuzzy PID-based Rolling State Prediction}

Considering the heavy burden of calculation in the online optimization in conventional nonlinear model predictive control, we present a rolling state prediction process with lower computations to replace the rolling optimization based on the complex nonlinear quadratic programming. This rolling state prediction makes the controller predict a series of states of system in the future by means of fuzzy PID control and enables the control signal in each sample contains the information about the future dynamics of the system, thus offsetting the deficiency of lacking future prediction capability in conventional controllers. The steps of rolling state prediction at sample time $\mathrm{k}$ is explained as follows: 
(1) Update current PID parameters $K_{p}(\mathrm{k}), K_{i}(\mathrm{k}), K_{d}(\mathrm{k})$ with the utility of the principle of fuzzy PID in Section 2.1.

(2) Predict the states at next sample time $\hat{\mathbf{X}}(\mathrm{k}+1 \mid \mathrm{k})$ on the basis of the current state $\mathbf{X}(\mathrm{k})$ and the predicted PID control movement $\Delta u(\mathrm{k} \mid \mathrm{k})$ by substituting $\hat{\mathbf{X}}(\mathrm{k}+1 \mid \mathrm{k})$ and $u(\mathrm{k} \mid \mathrm{k})$ for $\mathbf{X}(\mathrm{k}+1)$ and $u(\mathrm{k})$ in Equation (1), respectively. The above control input $u(\mathrm{k} \mid \mathrm{k})$ satisfies $u(\mathrm{k} \mid \mathrm{k})=u(\mathrm{k}-1)+\Delta u(\mathrm{k} \mid \mathrm{k})$.

(3) Assume $i=1$, Updating the PID predictive parameters $K_{p}(\mathrm{k}+\mathrm{i} \mid \mathrm{k}), K_{i}(\mathrm{k}+\mathrm{i} \mid \mathrm{k}), K_{d}(\mathrm{k}+\mathrm{i} \mid \mathrm{k})$ by repeating step (1) with the last prediction information. Then update $i=i+1$ and repeat the above operations in step (3) until $i$ reaches $N_{p}-1$, where $N_{p}$ is the prediction horizon.

(4) Record all the predictive control movement $\Delta u(\mathrm{k}+\mathrm{i}), \mathrm{i}=0,1, \cdots, N_{p}$ within the prediction horizon as a control sequence vector $\mathbf{u}(\mathrm{k})$.

The flow chart of the fuzzy PID-based rolling state prediction is illustrated in Figure 1.

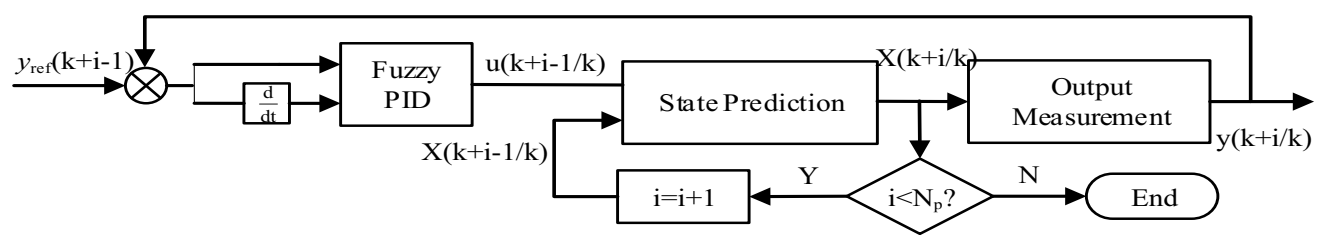

Figure 1. Flow chart of the fuzzy PID-based rolling state prediction

\subsection{Real-time Control Movement Integration}

The real-time control movement in this paper not only contains the first element of the predictive control sequence but also the rest of them, which differs from ordinary MPC methods. We introduce the concept of the level of confidence to its solution. The confidence probability of each element in the control movement sequence attenuates exponentially with the number of samples away from present. The computational formula of the real-time control movement is stated in Equation (2).

$$
\Delta u(k)=\sum_{i=0}^{N_{p}-1} \alpha^{i} \cdot \Delta u(\mathrm{k}+\mathrm{i} \mid \mathrm{k}), \quad i=0,1, \cdots, \mathrm{N}_{p}-1
$$

\section{Nonlinear Model for Excitation System}

The excitation system is composed of the controller, the power system stabilizer (PSS), the exciter (or the amplifier) and the generator [8], as shown in Figure 2.

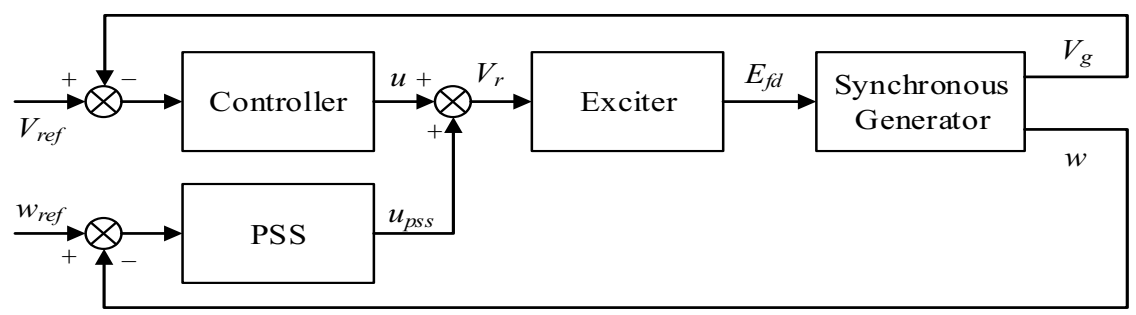

Figure 2. Block diagram of the excitation system

\subsection{Power System Stabilizer and Exciter}


Power system stabilizer (PSS) is added as an auxiliary control signal with which the damping property of the system is enhanced [9]. In this paper, the model of PSS is stated in Equation (3).

$$
\frac{u_{p s s}(\mathrm{~s})}{\omega_{r e f}(\mathrm{~s})-\omega(\mathrm{s})}=\frac{T_{w} \mathrm{~s}}{1+T_{w} \mathrm{~s}} \cdot \frac{1+T_{1} \mathrm{~s}}{1+T_{2} \mathrm{~s}} \cdot \frac{1+T_{3} \mathrm{~s}}{1+T_{4} \mathrm{~s}}
$$

where, $u_{p s s}$ is the output of PSS, $\omega_{r e f}$ is the given rotor angular speed, $\omega$ is the measured rotor angular speed, $T_{w}$ is time constant of PSS and $T_{1(2 / 3 / 4)}$ are lead-lag time constant.

The exciter in this paper can also be viewed as an amplifier consist of a gain element and a time constant since the excitation voltage $u_{f}$ and the EMF $E_{f d}$ is simplified to be the same here. Hence, the transfer function model of the exciter is stated in Equation (4).

$$
\frac{E_{f d}(\mathrm{~s})}{V_{r}(\mathrm{~s})}=\frac{K_{a}}{T_{a} s+1}
$$

where, $V_{r}$ is the control signal, $K_{a}$ is the gain of the exciter and $T_{a}$ is the constant of the exciter.

\subsection{Synchronous Generator and Its Connected Power System}

The model of the studied power system consists of a generator linked to an infinite bus through paralleled transmission lines can be expressed in the form of nonlinear state-space equations as shown in Equation (5) [10]. It is apparently in the form of an affine nonlinear system stated in Equation (1).

$$
\left\{\begin{array}{l}
T_{d 0}^{\prime} \frac{d E_{q}^{\prime}}{d t}=E_{f}-E_{q}^{\prime}-\left(X_{d}-X_{d}^{\prime}\right) \frac{U \sin \delta}{X_{q \Sigma}} \\
T_{j} \frac{d \omega}{d t}=P_{m}-\left(\frac{E_{q}^{\prime} U}{X_{d \Sigma}^{\prime}} \sin \delta+\frac{U^{2}\left(X_{d \Sigma}^{\prime}-X_{q \Sigma}\right)}{2 X_{d \Sigma}^{\prime} X_{q \Sigma}} \sin 2 \delta\right)-D(\omega-1) \omega_{0} \\
\frac{d \delta}{d t}=(\omega-1) \omega_{0}
\end{array}\right.
$$

where, $U$ is the constant voltage of the infinite bus, $\delta$ is the rotor angle of the generator, $E_{q}^{\prime}$ is the transient potential of q axis, $T_{d 0}^{\prime}$ is the transient time constant of d axis, $T_{j}$ is the inertial coefficient of the generator, $X_{d}^{\prime}$ and $X_{q}$ are transient potential of $\mathrm{d}$ axis and synchronous potential of $\mathrm{q}$ axis, respectively. $X_{d \Sigma}^{\prime}$ and $X_{q \Sigma}$ is calculated by $X_{d \Sigma}^{\prime}=X_{d}^{\prime}+X_{T L}$ and $X_{q \Sigma}=X_{q}+X_{T L}$ in which $X_{T L}$ is the total equivalent reactance on the transmission line. The continuous state-space equations is discretized by fourth-order Runge- Kutta method in the numerical simulation.

\section{Numerical Simulations}

To verify the effectiveness of the application of the proposed fuzzy predictive PID control method in the excitation system of the synchronous generator, simulations have been conducted in a power system in which a single machine connected to an infinite bus with its initial steady state given: Parameters of the generator are $X_{d}=0.964, X_{q}=0.578, X_{d}^{\prime}=0.238, D=0.10, T_{d 0}^{\prime}=5.43, T_{j}=9.28$.

Parameters of the bus and transmission lines are $X_{T}=0.125, X_{L}=0.576, U=1.00$.

Parameters of the exciter and the PSS are set as $K_{a}=50, T_{a}=0.005, K_{p s s}=0.1, T_{w}=5$.

The steady state before the disturbances happen (namely the initial state) in the simulations is $P_{0}=1.00, \delta_{0}=38.946, \omega_{0}=1.00$. All simulations is running under MATLAB R2014a environment. 


\subsection{Terminal Voltage Step Case}

A 5\% step is added on the terminal voltage of the generator at 1 second in the simulation. The FPPID control scheme is compared with the conventional PID and fuzzy PID control scheme. Simulation results viewed in Figure 3 show the significant enhancement in the calm down of the terminal voltage disturbance. Slight extra damping ability is also brought by FPPID in comparison with the other two controls. Note that in this paper, damping property of the excitation system is mainly decided by PSS auxiliary signal and all three controller in the experiment have already been equipped with a conventional PSS of which the transfer function is stated in Equation (3).
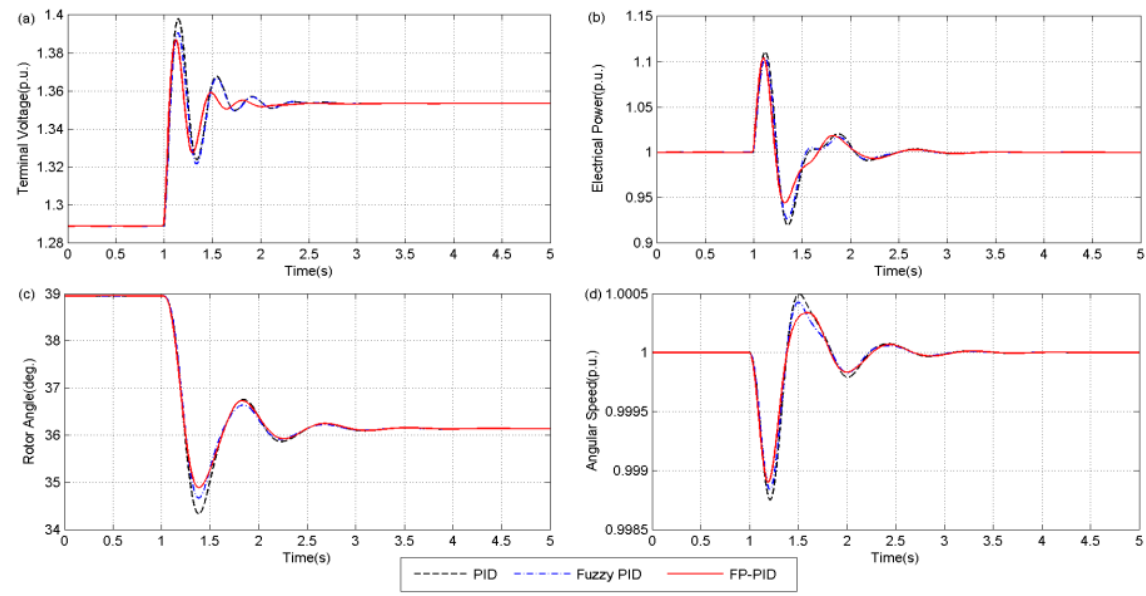

Figure 3. Response of the terminal voltage step of the synchronous generator

\subsection{Three-phase Short Circuit Case}

In this experiment, a three-phase short circuit fault on the high-voltage side of one of the paralleled transmission lines is taking place at 1 second. The faulted line is cut off within 0.2 second after the fault occurs. As shown in Figure 4, simulation results show the better voltage-tracking capability of the FP-PID over the comparative control methods with lower overshoot and less setting time compared with the conventional PID and fuzzy PID controls. It verifies the voltage recovery ability of the controller in serious disturbance situations, which is the most significant function of the excitation control.
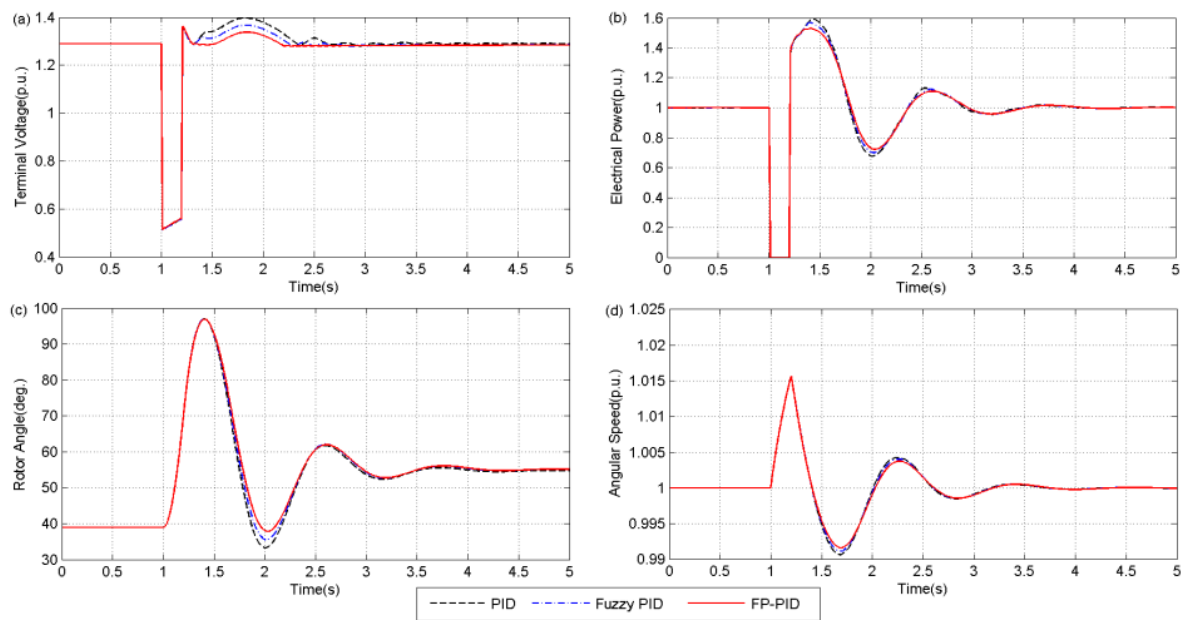

Figure 4. Response of the three-phase short circuit fault on transmission line 


\section{Conclusions}

In this paper, a modified fuzzy predictive PID (FP-PID) control scheme in the synchronous generator excitation system is proposed to enhance the voltage-tracking performance and decrease the computational burden. The fuzzy PID control theory have been utilized in rolling state prediction to replace the conventional rolling optimization process in every sampling instance due to its low calculated amount and control parameter adaptation characteristic. The concept of the confidence level is introduced to the solution of the real-time control movement, which makes it possible for the control signal contains the future dynamic information of the system. Numerical simulations under different disturbance conditions have been conducted using the proposed control method as well as the comparative ones. Experimental results have demonstrated the superiority of the proposed FP-PID control over conventional PID and fuzzy PID controls in control performances.

\section{Acknowledgement}

This work was supported by the Key National Natural Science Foundation of China (No. 51239004).

\section{References}

1. V Mukherjee, S.P. Ghoshal, Electr. Pow. Syst. Res. 77, 12 (2007)

2. G. Shabib, Ain Shams Eng. J. 3,2 (2012)

3. S. Pillutla, A. Keyhani, Int. J. Elec. Power. 19, 6 (1997)

4. J. Fraile-Ardanuy, P.J. Zufiria, Neurocomputing. 70, 16 (2007)

5. R. Zhang, S. Wu, R. Lu, et al., Chemometr. Intell. Lab. 135 (2014)

6. T. Sato, Control Eng. Pract. 18, 2 (2010)

7. K. Aboudolas, M. Papageorgiou, A. Kouvelas, et al., Transport. Res. C-Emer. 18, 5 (2010)

8. P. Kundur, Power system stability and control (McGraw-Hill, NewYork, 1994)

9. E.S. Ali, Int. J. Elec. Power. 61 (2014)

10. Y. Wan, B. Jiang, Automatica. 62 (2015) 